\title{
Plasma Fluctuations Could Generate Bright Pulsar Emission
}

\section{Calculations link coherent pulsar emission to fluctuations in electron-positron production.}

\section{by Alice K. Harding ${ }^{1}$}

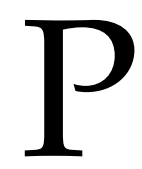
ulsars-neutron stars spinning up to hundreds of times a second-emit radio-frequency beams that seem impossibly bright. The laws of physics require that such bright pulses be produced by a coherent radiation mechanism, in which groups of particles emit in sync with each other, like the atoms that fire in unison to produce laser light. Despite much research effort since pulsars were discovered, deciphering this mechanism has been stubbornly elusive. A study by Alexander Philippov of the Flatiron Institute in New York and colleagues, details a promising new proposal for explaining this coherent emission [1]. Their calculations-so far carried out in a simplified 2D case-suggest that radiation with desired co-

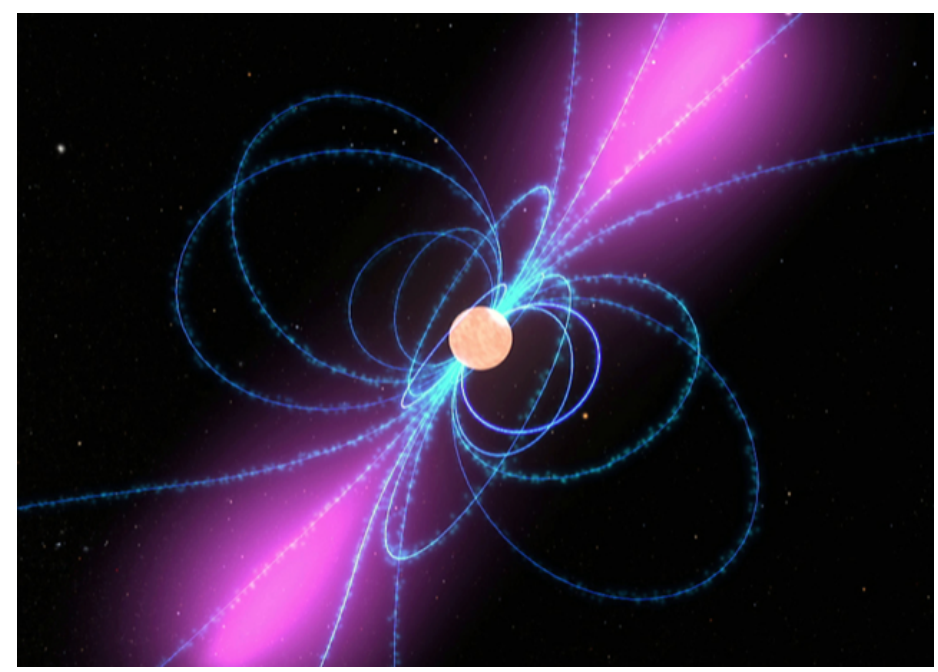

Figure 1: Charged particles (blue) moving along the lines of a pulsar's magnetic field produce beams of coherent radiation (purple). Philippov et al. suggest that this radiation is produced by fluctuations in the process that generates electron-positron pairs in the pulsar. (NASA)

${ }^{1}$ Astrophysics Science Division, NASA Goddard Space Flight Center, Greenbelt, MD, USA herence properties can be produced via a fluctuating process that generates electron-positron pairs near the pulsar.

By cosmic standards, pulsars are tiny objects-about the size of Manhattan. But they possess the strongest known magnetic fields in the Universe. The supernova explosions from which they originate leave the pulsars rotating at high speed. This rotation powers the emission of beams of electromagnetic radiation out of the pulsar's magnetic poles. Such beams are emitted steadily but sweep in and out of our line of sight like lighthouse lamps. The emitted spectrum spans a very large range of frequencies, from gamma rays to radio waves. The radio component allowed the first discovery of a pulsar by a radio telescope in 1967 [2] and thousands of radio pulsars since.

If pulsars were incoherent blackbody emitters, their radio brightness would imply an unrealistic temperature of $10^{25}-10^{30} \mathrm{~K}$. Thus the emission must be coherent. Coherent emission of radio waves, albeit with much lower brightness, is seen to come from the Sun and from the magnetospheres of Earth and Jupiter [3] and is well studied in laboratory experiments [4]. In astrophysical settings, this emission typically involves plasmas. Coherence can be generated when the plasma's charged particles get bunched up along the same trajectories in space or with the same momenta. It can also be produced if the particles have an inverted population, in which more particles are in excited energy states than in lower, unexcited states. This population inversion is the mechanism allowing a maser (the microwave analog of a laser) or a laser to produce coherent stimulated emission.

It is unclear what coherent processes operate in pulsars. Pulsar plasma is different from solar plasma, as we believe that it's made up of electrons and antielectrons (positrons) rather than electrons and ions. This electron-positron plasma comes from pair production of energetic photons, which are emitted by particles accelerated in the electric field induced by the rotating magnetic field of the pulsar. In a pulsar, photons can have sufficient energy, and the magnetic field can be sufficiently strong for this pair production mechanism to be very efficient [5]. This process can trigger cascades-photons produce pairs that radiate more photons that produce more pairs, leading to dense plasmas [6, 7]. The produced pairs move along the lines of the magnetic field at relativistic speeds with a large spread of velocities (Fig. 1) 
and are unevenly distributed in space.

Many theories have been proposed to explain how this kind of plasma can lead to the observed pulsar emission, but they all have fundamental problems [3]. Certain instabilities could bunch the particles along curved magnetic field lines, leading to "coherent curvature emission." However, this mechanism produces frequencies that are much lower than observations, and coherent bunches would require pairs whose energy spread is much smaller than that expected for pairs in the pulsar [8]. Another explanation involves a relativistic version of the beam instabilities that operate in solar radio bursts. But in pulsars these instabilities grow too slowly, and the pair energy spread is again too large for this mechanism to be effective. A third idea involves synchrotron maser emission but requires special circumstances that are not satisfied in all pulsars.

In their model, Philippov and colleagues exploit a new idea recently introduced by co-author Andrey Timokhin [9]; namely, that pulsar pair cascades may not occur steadily, as was assumed for many decades, but in submillisecondlong cycles. Simulating pair cascades in 1D, Timokhin found that, as particles are accelerated by the electric field and emit high-energy photons, they produce a burst of electronpositron pairs that completely screen the electric field. This screening stops the acceleration and thus pair production. The field only remerges after the pairs escape the region, which takes a fraction of a millisecond at relativistic speeds. Timokhin also found that this periodic screening generated strong, superluminal electrostatic waves. In 1D, however, these waves cannot turn into propagating, coherent electromagnetic waves, as wave propagation requires electric-field components orthogonal to the magnetic field.

Extending the simulations to 2D, Philippov and colleagues modeled plasma dynamics over a larger region near the pulsar surface. They found that the electrostatic waves did indeed generate electromagnetic waves. This generation is possible because the electric field around pulsar polar caps isn't uniform, as it has to vanish at the boundary to the fully conducting region that's filled with plasma. The researchers showed that this geometry results in an oscillating electric field with a component perpendicular to the static magnetic field - what's needed to form an electromagnetic wave. Since this wave is generated by the collective motion of plasma, and not by the emission of individual particles, it is coherent. Thus the inherent pulsar mechanisms of pair production and electric-field screening can automatically produce coherent radio emission!

The researchers show that the spectra of the waves produced by this mechanism is consistent with that observed for pulsars. Their model, however, remains very much a "toy" model because it limits particle energies to values significantly lower than those of a real pulsar. As such, a number of questions remain open. Are the pair-induced waves sufficiently bright to match observations? Can they escape the pulsar's magnetosphere? These questions will only be answered by a 3D simulation of a larger region of space. Another issue requiring further investigation is the fact that this mechanism may be too efficient. According to the team's calculations, these waves could account for a large amount of the estimated energy loss that slows down pulsar rotation, but there is evidence that only a small fraction of this energy loss goes into pulsar radio emission. It's easy, however, to imagine several possible mechanisms that could reduce the power of the waves. It's also worth noting that the mechanism invoked by the researchers would not explain another type of radio emission, generated further away from the star, that's been seen in the famous Crab pulsar and in a few other pulsars.

Even with all these caveats, this result is a major step forward in the quest to uncover the pulsar radio emission mechanism. And, as Timokhin and colleagues suggested [10], the same mechanism could have broad implications, explaining, for instance, how even more highly magnetized neutron stars, called magnetars, produce the mysterious extragalactic fast radio bursts.

This research is published in Physical Review Letters.

\section{REFERENCES}

[1] A. Philippov et al., "Origin of pulsar radio emission," Phys. Rev. Lett. 124, 245101 (2020).

[2] A. Hewish et al., "Observation of a rapidly pulsating radio source," Nature 217, 709 (1968).

[3] D. B. Melrose, "Coherent emission mechanisms in astrophysical plasmas," Rev. Mod. Plasma Phys. 1, 5 (2017).

[4] N. Carlevaro et al., "Nonlinear physics and energetic particle transport features of the beam-plasma instability," J. Plasma Phys. 81, 5 (2015).

[5] T. Erber, "High-energy electromagnetic conversion processes in intense magnetic fields," Rev. Mod. Phys. 38, 626 (1966).

[6] J. K. Daugherty and A. K. Harding, "Electromagnetic cascades in pulsars," Astrophys. J. 252, 337 (1982).

[7] A. N. Timokhin and A. K. Harding, "On the polar cap cascade pair multiplicity of young pulsars," Astrophys. J. 810, 144 (2015).

[8] D. B. Melrose, "Maser pulse emission mechanisms," Pulsars: 13 Years of Research on Neutron Stars, Proceedings of IAU Symposium No. 95, edited by W. Sieber and R. Wielebinski (D. Reidel, Dordrecht, 1981), p. 133-139.

[9] A. N. Timokhin and J. Arons, "Current flow and pair creation at low altitude in rotation-powered pulsars' force-free magnetospheres: Space charge limited flow," Mon. Not. R. Astron. Soc. 429, 20 (2012).

[10] Z. Wadiasingh and A. Timokhin, "Repeating fast radio bursts from magnetars with low magnetospheric twist," Astrophys. J. 879, 4 (2019).

10.1103/Physics.13.96 